\title{
On chip immuno-affinity profiling of cancer- and benign hyperplasia-associated free prostate specific antigen
}

\author{
Maja M. Kosanovic ${ }^{\text {, Sanja R. Goc }}$, Goran S. Potpara ${ }^{\mathrm{b}}$ Miroslava M. Jankovic $^{\mathrm{a}, *}$ \\ ${ }^{a}$ University of Belgrade, Institute for the Application of Nuclear Energy, INEP, Zemun, Serbia \\ ${ }^{\mathrm{b}}$ Clinical Center Zemun, Department of Urology, Zemun, Serbia
}

\begin{abstract}
Prostate specific antigen (PSA) exhibits pronounced heterogeneity in both primary structure and glycan composition, resulting in the existence of different molecular forms. Investigation of PSA structure is a demanding task facing limitations due to inadequate sensitivity of analytical techniques and low concentrations of the different forms. This study aimed to profile free PSA (fPSA), especially lower molecular mass species lacking detailed classification, in normal seminal plasma and in sera from subjects with benign hyperplasia (BPH) or cancer of the prostate (PCa) as samples of known clinical relevance. fPSA forms were separated from complex proteomes on chips with immobilized anti-fPSA antibody followed by detection using surface-enhanced laser desorption/ionization time of flight mass spectrometry. At least 39 fPSA-immunoreactive species, ranging from 3-29 kDa were detected in seminal plasma. General fPSA profiles in seminal plasma and sera were similar, but differed in the abundance and presence of particular peaks/clusters of the lower molecular mass species. No striking difference in fPSA forms was observed between BPH and PCa samples, but some distinct peaks varied in intensity and frequency within or between groups. Obtained data verify fPSA heterogeneity that might be important for better exploration of all their molecular and marker potentials.
\end{abstract}

Keywords: Free PSA, molecular forms, prostate cancer, benign prostatic hyperplasia, SELDI-TOF/MS

\section{Introduction}

Prostate specific antigen (PSA), designated also as kallikrein $3(\mathrm{hKh} 3)$ is a serine protease, produced and secreted, predominantly, by the prostate gland [1,2]. In tissue, seminal fluid and urine, it is mainly present in the free form, whereas in serum, PSA is found in complex with extracellular protease inhibitors, such as $\alpha 1$-antichymotrypsin (ACT) or $\alpha 2$-macroglobulin (A2M) [3].

PSA is a $28.4 \mathrm{kDa}$ glycoprotein having $8 \%$ of carbohydrates in the form of a bianntenary N-linked oligosaccharide chain [4]. However, accumulated experimental evidence has revealed pronounced heterogeneity in both primary structure and glycan composition, result-

*Corresponding author. E-mail: miraj@inep.co.rs. ing in the existence of diverse isoforms whose ratio differs in normal physiology from that in cancer (PCa) or benign hyperplasia of the prostate (BPH) [5-16].

A catalog of molecular forms, established using PSA purified from human seminal plasma (SP), listed approximately twenty immunoreactive species ranging from 6-28 kDa. Generally, PSA from non-malignant sources showed a wider range of molecular masses than PSA of malignant origin [17]. Thus, the free form of PSA can comprise the precursor or zymogen form (proPSA), found to be elevated in cancer, as well as mature active/inactive and clipped forms. Among these BPSA with internal bond cleavage at Lys145 and Lys182 residues is supposed to be associated with BPH [14, 18,19]. In addition, heterogeneity in PSA glycan composition was also detected and seen as the existence of biantennary or monoantennary glycans, and/or various outer chain moieties and differently sialylated and fucosylated forms [10,13,20-25]. 
In the light of findings suggesting that some types of diversity are associated more frequently with cancer or benign hyperplasia of the prostate, it is plausible that the molecular structure of PSA may be more relevant for differential diagnosis of $\mathrm{BPH}$ and $\mathrm{PCa}$, than its serum concentration. Investigation of the structural properties of PSA is a very demanding task facing limitations due to intrinsic PCa heterogeneity, sensitivity and specificity of analytical techniques and the low concentration of PSA forms available for characterization. So far, different approaches have been made using single samples with high PSA concentrations, such as PCa sera or human seminal plasma, or pooled samples containing PSA concentrated by immunoadsorption or released from complexes in order to increase its abundance $[11,17,21,26,27]$. Lectin-based methods or mass spectrometry were employed for glycan characterization and different glyco/molecular forms were separated by chromatography, SDS-PAGE or 2D-PAGE, while PSA was usually detected and identified with polyclonal antibodies for total PSA [5,11,13,17,20,24,25, 28-32].

In this study we have addressed the issue of structural heterogeneity of PSA using a specific approach based on immunoaffinity capture in combination with mass spectrometry for detection of bound forms. Specifically, we aimed at profiling free PSA according to its reactivity with a monoclonal antibody of defined epitope reactivity, in seminal plasma and serum, as biological samples of known relevance in clinical use. The high resolution and sensitivity of detection enabled simple, one-step analysis of PSA forms directly from the samples, without any need for prior fractionation or modification. The results obtained revealed specific patterns of free PSA forms and gave more insight into low molecular species, so far lacking detailed classification.

\section{Materials and methods}

Monoclonal anti-free PSA antibody, isotype IgG2a, clone 8A6 (specific for conformational epitope I), was purchased from Hy Test (Turku, Finland). ProteinChip PS20 (preactivated surface) and sinapinic acid were from BioRad (Hercules, CA, USA). Total PSA and free PSA (fPSA) were determined using the appropriate IRMA PSA assays (INEP, Serbia) according to the manufacturer's instructions. The assays were calibrated against standards from the National Institute for Biological Standards and Control (Hertfordshire, United Kindgom): total PSA:PSA (90:10) first International standard NIBSC Code 96/670 and PSA (free) first International standard NIBSC Code 96/668. All other chemicals were reagent grade.

\subsection{Biological samples}

Samples of seminal plasma (SP) were obtained from consenting subjects seen at INEP-Zemun, Serbia, according to local ethical standards (document GSP/05; PR030/09) approved by Institutional committee of Institute for the Application of Nuclear Energy, INEP. Human semen samples had sperm parameters within the normal range according to the recommended criteria of the World Health Organization 1999 [33]: sperm volume $>2 \mathrm{~mL}$, liquefaction $<30 \mathrm{~min}$, total number of spermatozoa $>20 \times 10^{6}$, number of spermatozoa with normal morphology $>70 \%$ and total number of mobile spermatozoa $>50 \%$. Samples were allowed to liquefy (without the addition of protease inhibitors), after which the sperm cells and other debris were removed from the ejaculate by centrifugation at $900 \mathrm{x} \mathrm{g}$ for $20 \mathrm{~min}$. The pool was formed from ten samples and was used immediately or stored at $-20^{\circ} \mathrm{C}$ until processed.

Archive specimens of individual sera from BPH and PCa patients (15 each) seen for PSA determination as part of follow up were examined. Mean age was 63 years in the BPH group (range 56 to 70 ) and 67 years in the PCa group (range 58 to 78 ). Diagnoses were confirmed using clinically established protocols based on PSA level, physical examination, ultrasound and biopsy. Samples of PCa sera were from patients diagnosed with locally advanced and advanced cancers. PSA concentrations ranged from $0.4-6.8 \mu \mathrm{g} / \mathrm{L}$ and $48.1-$ $255 \mu \mathrm{g} / \mathrm{L}$ for individual BPH and PCa serum samples, respectively. Sera were used immediately or stored in aliquots at $-20^{\circ} \mathrm{C}$ until processed.

\subsection{PSA profiling on ProteinChip array-immobilized anti-fPSA antibody}

Anti-fPSA monoclonal antibody $(5 \mu \mathrm{L})$ was applied to each spot of the preactivated-surface (PS20) protein chip array, and incubated in a humid chamber overnight at $4^{\circ} \mathrm{C}$. The spots were washed thrice with $5 \mu \mathrm{L}$ of $0.05 \mathrm{M}$ PBS, $\mathrm{pH} 7.2$ for $1 \mathrm{~min}$ at room temperature (RT) and then blocked with $0.5 \mathrm{M}$ Tris-HCl, $\mathrm{pH} 8.0$ buffer for 1 hour in a humid chamber at RT. After washing thrice using the same procedure, $5 \mu \mathrm{L}$ of sample (SP or serum) was added to each spot and incubated for 2 hours in a humid chamber at RT. The spots were 
rinsed again thrice with $0.05 \mathrm{M}$ PBS, $\mathrm{pH} 7.2$, and twice with deionized water. All procedures (antibody coupling, washing steps, blocking and sample binding), included shaking (150 rpm). After drying, $1 \mu \mathrm{L}$ of $50 \%$ sinapinic acid (in acetonitrile/dH2O/trifluoroacetic acid [50\%/49.9\%/0.1\%]) was added to each spot, dried and then reapplied.

Protein chips were analyzed by SELDI-TOF/MS [34] using the ProteinChip Reader, Series 4000, Personal edition (BioRad). All spectra were acquired in $25 \mathrm{kV}$ positive ion acquisition mode, mass range $2.5-50 \mathrm{kDa}$ and with 8815 laser shots per spot. The laser energy was 6000 nJ. Mass was calibrated with ProteinChip all-in-one protein standards II. All spectra were analyzed using Ciphergen Express Software 3.0 (BioRad, Hercules, CA, USA).

\subsection{Analytical characteristics of on-chip immuno-affinity capture of fPSA}

Serial dilutions of PCa serum samples (fPSA $23.2 \mu \mathrm{g} / \mathrm{L})$ were placed on chip immobilized anti-free PSA antibody and processed as described. The lowest fPSA concentration giving the characteristic cluster of peaks in the $26-29 \mathrm{kDa}$ region with signal to noise ratio $>10$ (arbitrary) was defined as the detection limit. The linearity of the method was evaluated according to the area under the peak vs the concentration curve.

In order to assess non-specific binding, two types of controls were used: 0.05 M PBS, pH 7.2, or PSAdepleted serum instead of serum samples.

\section{Results}

The experimental system for the qualitative analysis of fPSA forms was based on direct immunoaffinity capture without any pre-treatment of the samples, similarly as used for determination of fPSA concentration by immunoassay. The analytical characteristics of this system i.e. good linearity (Fig. 1A) as assessed from the area under the peak vs the concentration curve, and a detection limit of $0.046 \mu \mathrm{g} / \mathrm{L}$, as estimated from the profiles of intact fPSA (Fig. 1B), allowed reliable analysis of samples containing a broad concentration range, as found in SP, and BPH or PCa sera.

Profiles of the molecular forms of fPSA, on protein chips with immobilized monoclonal anti-fPSA antibody specific for epitope I are shown in Figs 2 and 3. Samples generally gave patterns comprising distinct forms with molecular masses corresponding to that of
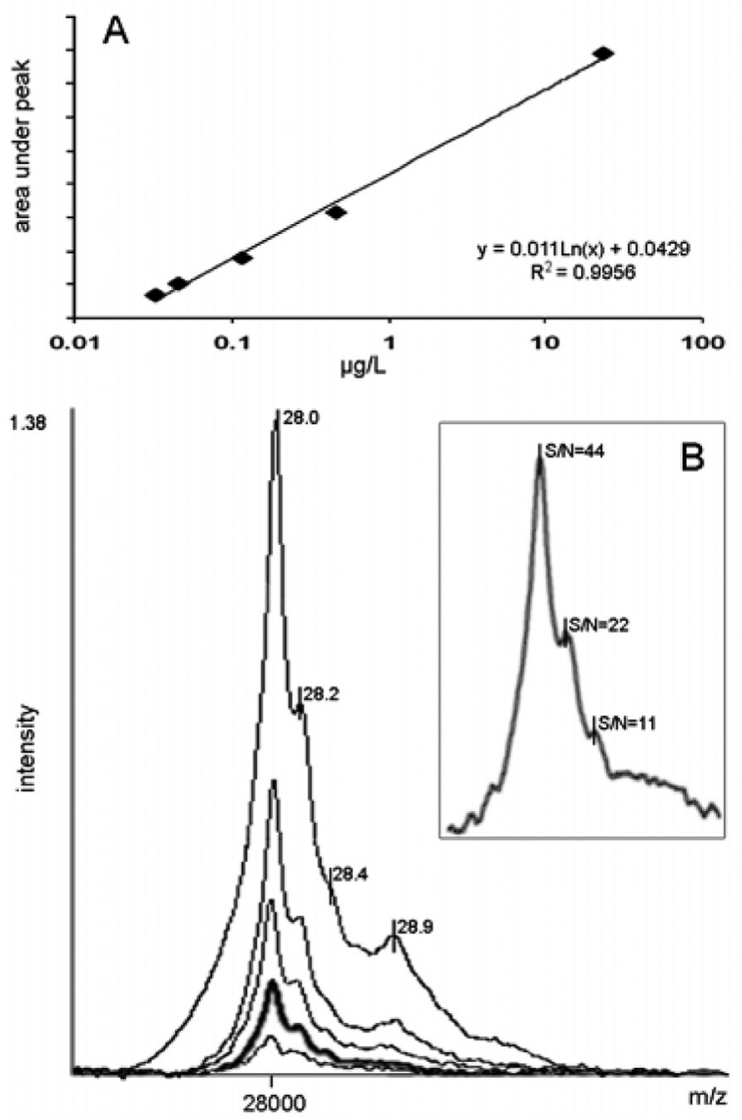

Fig. 1. Analytical characteristics of on-chip immuno-affinity capture of fPSA. A. Linearity: mass spectra of intact fPSA forms separated from PCa serum (fPSA, $23.2 \mu \mathrm{g} / \mathrm{L}$ ) diluted to concentrations of $0.464 ; 0.116 ; 0.046$ and $0.003 \mu \mathrm{g} / \mathrm{L}$. Dilution experiments demonstrated the linearity of on-chip immuno-affinity capture of fPSA (the correlation coefficient of 0.9956). B. Detection limit: mass spectrum of intact fPSA forms separated from the same PCa serum at $0.046 \mu \mathrm{g} / \mathrm{L}$. The detection limit was defined as the lowest concentration of fPSA giving the characteristic cluster of peaks with a signal to noise ratio $>10$ (arbitrary) at $26-29 \mathrm{kDa}$. On-chip immunoaffinity-captured molecules were detected by SELDI-TOF mass spectrometry in ion-positive mode at $25 \mathrm{kV}$ and analyzed using ChipergenExpress Software 3.0 (BioRad, Hercules, CA, USA). Calibration was performed with ProteinChip all-in-one protein standards II.

intact fPSA (Fig. 2) and its known fragments generated by proteolytic degradation (Fig. 3). The general patterns of fPSA from SP and sera were similar, but differed in the intensity or presence of particular peaks within clusters. Thus, in SP, at least five molecular species differing in relative abundance in the 26$29 \mathrm{kDa}$ region were detected (Fig. 2). A comparable profile was obtained with PSA standard preparations (NIBSC Code 96/668), which also originated from SP, but represent a purified preparation. The fPSA profiles from $\mathrm{PCa}$ and $\mathrm{BPH}$ were similar, comprising fPSA 


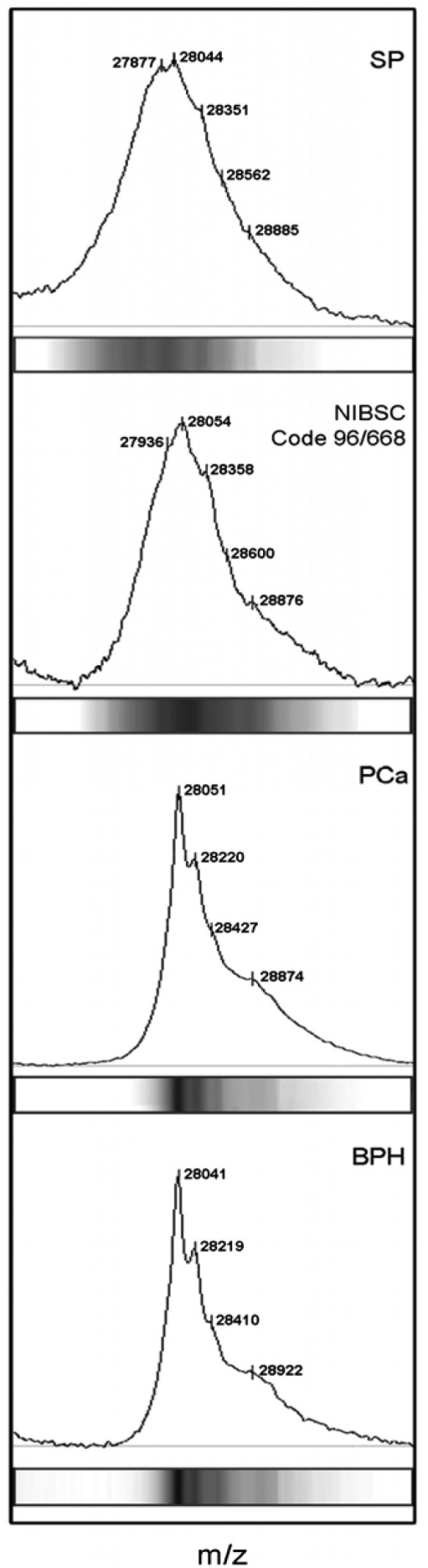

Fig. 2. Representative SELDI-TOF spectra of intact fPSA forms. Anti-fPSA monoclonal antibody was immobilized on PS20 protein chip array and incubated with corresponding samples for 2 hours in a humid chamber at RT. The spots were rinsed and after drying, 50\% sinapinic acid was added to each spot, dried and then reapplied. The spectra were obtained by SELDI-TOF/MS. Mass spectra and gel views of the cluster of fPSA peaks in the 26-29 kDa region. SP - normal seminal plasma, NIBSC Code 96/668 - PSA (free) first International standard, PCa - prostate cancer serum and $\mathrm{BPH}$ - benign prostatic hyperplasia serum. In the spectra view, the $\mathrm{x}$-axis represents the distribution of ions by mass (mass to charge ratio, $\mathrm{m} / \mathrm{z}$ ) and the $\mathrm{y}$-axis represents signal intensity of the ions. In the gel view, the color intensity of the bands corresponds to peak height. 


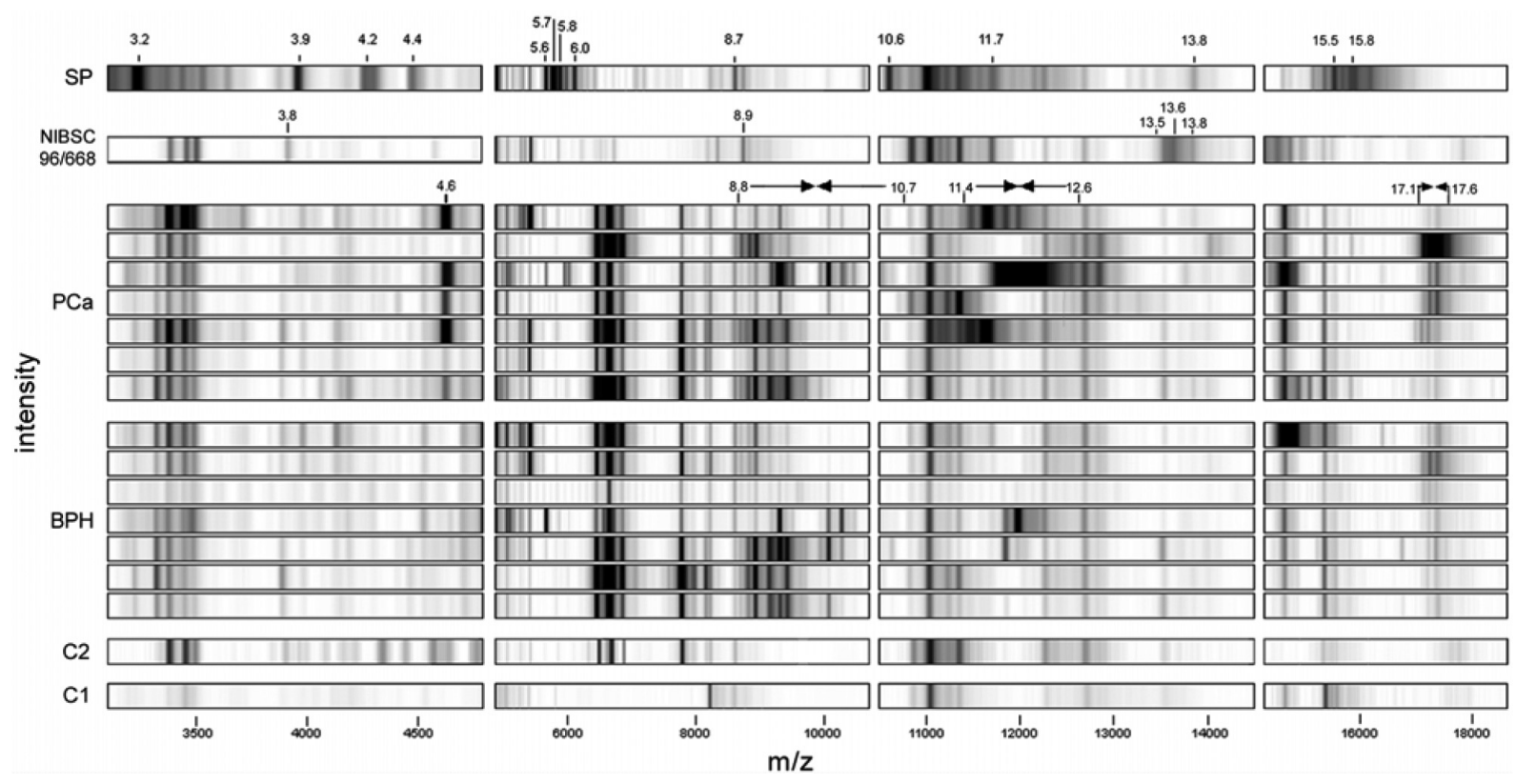

Fig. 3. SELDI-TOF spectra of lower molecular mass fPSA-immunoreactive forms. Representative SELDI-TOF MS spectra (gel view) are shown as separate mass regions $(3-5 ; 5-10,10-14$; and 14-19), with Mw designated on x-axes. SP - human seminal plasma; NBSCI code 96/668 fPSA standard preparation; PCa-prostate cancer sera; BPH-benign hyperplasia sera. C1- control sample, no serum added; C2- control sample, PSA depleted-serum. Peaks found on the control chips were considered as non-specific.

immunoreactive species in the $28-29 \mathrm{kDa}$ range, but missing peaks in the $26-28 \mathrm{kDa}$ region.

Immunoreactive species of lower molecular mass were detected in human SP at 3.2, 3.9, 4.2, 4.4, 5.7, 8.7, 10.6, 11.7, 13.8 and $15.7 \mathrm{kDa}$ (Fig. 3). In addition, a series of lower intensity peaks was also found (total number of peaks $=34$ ). Immunoreactive fPSA in the 5.6-6.0 $\mathrm{kDa}$ and 15.5-16.1 kDa regions appeared as clusters of several peaks indicating prominent heterogeneity. In the spectrum of the fPSA standard preparation the number of immunoreactive species was lower than in the SP spectrum and they were found at 3.8, 8.8 $11.7 \mathrm{kDa}$ and in the 13.5-13.9 $\mathrm{kDa}$ region.

In the BPH- and PCa-serum samples, the fPSAimmunoreactive peaks were similar and detected at $3.2 \mathrm{kDa}$, in the 8.8-10.7 and 11.4-12.6 kDa regions and as a cluster at 17.1-17.6 kDa (Fig. 3). Intrinsic heterogeneity among the samples was reflected in the corresponding mass spectra, indicating qualitative differences seen as frequencies of peaks/clusters found for each group of samples, and also quantitative differences seen as peak intensities, possibly reflecting different PSA concentrations in each sample within or between groups. However, no significant difference was observed except for a distinct and intense peak at $4.6 \mathrm{kDa}$ found in PCa samples (12/15).

\section{Discussion}

The reported data on proteomic patterns of fPSA obtained by immunoaffinity capture verify its heterogeneity and update existing information on molecular subforms and their fragments in relation to different patho/biological sources. The PSA examined, actually corresponded to the fraction whose concentration is determined in the laboratory test for free PSA, and differed from fractions involving antigen liberated from complexes or antigen purified using polyclonal or monoclonal anti-total PSA antibodies, as used in several related studies.

The predicted molecular mass of PSA calculated from the consensus sequence for the peptide portion of the molecule is $\mathrm{Mr}$ 26,079 $\mathrm{Da}$, and the calculated molecular mass of its carbohydrate structure is $\mathrm{Mr}$ 2,3518 $\mathrm{Da}$ [4]. However, it is known that, during biosynthesis and processing, several PSA forms can be generated and that lower molecular mass forms (4$22 \mathrm{kDa}$ ) are mainly produced by proteolytic/internal cleavage or alternative splicing [35].

A catalog of SP PSA forms, established by 2-DE of a chromatographically purified sample, comprised approximately thirty spots among which twenty contained PSA peptide fragments. They were classified according to molecular mass into groups of glycosylated 
forms: gp28, gp22, gp18, gp12 and non-glycosylated forms: p26, p20, p16, p10 and p6 [17].

Under our experimental conditions, at the position corresponding to intact PSA, one cluster of differently abundant peaks was detected: major 27,877; 28,044; 28,351 and minor 28,562 and 28,885 Da in the SP sample. We also succeeded in distinguishing lower molecular mass PSA forms in detail and obtained comprehensive insight, taking advantage of detection by mass spectrometry rather than the commonly used 2-DE immunodetection. Thus, out of the total of 34 , ten main lower molecular mass species were observed in the 3$20 \mathrm{kDa}$ region, differing in heterogeneity within particular mass clusters. Among them, distinct clusters of PSA species at 5.6-6.0 kDa and 15.5-16.1 kDa showed pronounced variability.

Our results gave estimated masses that can be correlated to reference data indicating that the purified SP PSA standard preparation contains two PSA isoforms, a minor one of Mr 28,284 and a major one of 28,430, as measured by ion spray mass spectrometry [4]. The existence of intact SP PSA forms (at least five isoforms) is supposed to be related to differences in their sugar composition, although the influence of other structural modifications cannot be ruled out $[5,8,18,23,26]$. The same may hold true for our observation on the variability of the detected PSA subforms. Our results for the PSA standard preparation gave the same profile for intact PSA as seminal plasma but differences in smaller molecular forms.

In addition to SP, fPSA subforms were examined in BPH- and PCa-sera. Existing experimental data suggest distinct structural properties concerning glycosylation in comparison with SP PSA and also differences in composition regarding the presence or not of particular PSA forms. Comparison of SP PSA with both BPH PSA and PCa PSA, indicated the main differences to be the absence/decrease of the major peak at 27,877 and peaks in the $3.2-6.0 \mathrm{kDa}$ region. When considering these results one should bear in mind that the experimental design using antibodies for free PSA allowed only detection of uncomplexed PSA, and that the difference found may be partly related to known compositions of examined samples in respect to the ratio of enzymatically active/inactive or precursor PSA forms. Thus, some evidence, which can be connected with these prepositions, indicates that the molecular mass of PSA liberated from the complex with ACT is 27,755 Da and that uncomplexed fPSA isolated from PCa sera is larger than SP PSA [34,37].

Accumulated data concerning the heterogeneity of fPSA in serum samples point to several isoforms of intact PSA with presumed differences in glycosylation (mainly sialylation and fucosylation) [22,38]. Howev$\mathrm{er}$, there is evidence indicating at least 15 immunoreactive serum-derived fPSA spots of different molecular mass or charge, among which those forms with molecular masses less than $30 \mathrm{kDa}$ are present in a higher percentage in $\mathrm{BPH}-$ than in $\mathrm{PCa}-$ serum, or some are completely absent from PCa [11]. As for low molecular mass serum fPSA, our results contradict the finding about their predominance in BPH compared to PCa. Moreover, this study indicated similarity in molecular forms of serum fPSA of different origin. Namely, there were no striking differences between BPH PSA and PCa PSA patterns.

A study on glycan composition of fPSA subforms suggested that there may be no difference in processing and accumulation of PSA in cancerous and noncancerous prostate tissue [24]. Generally, due to the nature and complexity of PCa, PSA molecules originating from normal, inflamed and neoplastic tissue exist simultaneously in serum and other biological fluids, excluding exclusive selectivity concerning the source of antigen production. However, moderate changes in the ratio of particular PSA isoforms can be characteristically associated with BPH or PCa [5,6]. Thus, detailed inspection of spectrum profiles revealed some specificities related to the frequency of the presence of particular species or clusters of peaks in BPH PSA compared to PCa PSA. The most telling example refers to a $4.6 \mathrm{kDa}$ species found with high frequency only in PCa samples.

Since this study is an experimental one, aiming at a new experimental approach for gaining more insight into basic aspects of fPSA forms, a clinical evaluation in terms of comparison of BPH PSA and PCa PSA profiles was beyond its scope. However this finding might deserve attention as a tool to complement current approaches in analytical considerations of PSA measurements. They direct further investigations to use this experimental system, in our experience a most simple and effective one, to run a larger series of samples for validation of possible differences or to complement the existing data with PSA forms reactive with other available capturing molecules.

In conclusions, this study reports a simple, sensitive, specific, one-step analysis of fPSA forms, without the need for any pretreatment of the sample examined. The results obtained confirmed the importance of determining the proteomic pattern of heterogeneous analytes, such as PSA, for better understanding and exploration of all their molecular potentials. 


\section{Acknowledgment}

This work is supported by the Ministry of Education and Science of the Republic of Serbia, project code 173010.

\section{References}

[1] C. Diamandis, G.M. Yousef, L. Luo, A. Magklara and C.V. Obiezu, The new human kallikrein gene family: implications in carcinogenesis, Trends Endocrinol Metab 11 (2000), 54-60.

[2] C.K. Gjertson and P.C. Albertsen, Use and assessment of PSA in prostate cancer, Med Clin North Am 95 (2011), 191-200.

[3] H. Lilja, A. Christensson, U. Dahlén, M.T. Matikainen, O. Nilsson, K. Pettersson and T. Lövgren, Prostate-specific antigen in serum occurs predominantly in complex with alpha 1-antichymotrypsin, Clin Chem 37 (1991), 1618-1625.

[4] A. Belanger, H. Halbeek, H.C. Graves, K. Grandbois, T.A. Stamey, L. Huang, I. Poppe and F. Labrie, Molecular mass and carbohydrate structure of prostate-specific antigen: studies for establishment of an international PSA standard, Prostate 27 (1995), 187-197.

[5] P.R. Huber, H.P. Schmid, G. Mattarelli, B. Strittmatter, G.J. van Steenbrugge and A. Maurer, Serum free prostate specific antigen: isoenzymes in benign hyperplasia and cancer of the prostate, Prostate 27 (1995), 212-219.

[6] H. Hilz, J. Noldus, P. Hammerer, F. Buck, M. Lück and H. Huland, Molecular heterogeneity of free PSA in sera of patients with benign and malignant prostate tumors, Eur Urol 36 (1999), 286-292.

[7] S.D. Mikolajczyk, L.S. Millar, K.M. Marker, T.J. Wang, H.G. Rittenhouse, L.S. Marks and K.M. Slawin, Seminal plasma contains BPSA, a molecular form of prostate-specific antigen that is associated with benign prostatic hyperplasia, Prostate 45 (2000), 271-276.

[8] A. David, N. Mabjeesh, I. Azar, S. Biton, S. Engel, J. Bernstein, J. Romano, Y. Avidor, T. Waks, Z. Eshhar, S.Z. Langer, B. Lifschitz-Mercer, H. Matzkin, G. Rotman, A. Toporik, K. Savitsky and L. Mintz, Unusual alternative splicing within the human kallikrein genes KLK2 and KLK3 gives rise to novel prostate-specific proteins, J Biol Chem 277 (2002), 1808418090.

[9] M.B. Gretzer and A.W. Partin, PSA markers in prostate cancer detection, Urol Clin North Am 30 (2003), 677-686.

[10] R. Peracaula, G. Tabarés, L. Royle, D.J. Harvey, R.A. Dwek, P.M. Rudd and R. de Llorens, Altered glycosylation pattern allows the distinction between prostate-specific antigen (PSA) from normal and tumor origins, Glycobiology 13 (2003), 457470.

[11] K. Jung, J. Reiche, A. Boehme, C. Stephan, S.A. Loening and D. Schnorr, Analysis of subforms of free prostate-specific antigen in serum by two-dimensional gel electrophoresis: potential to improve diagnosis of prostate cancer, Clin Chem $\mathbf{5 0}$ (2004), 2292-2301.

[12] S.D. Mikolajczyk and H.G. Rittenhouse, Tumor-associated forms of prostate specific antigen improve the discrimination of prostate cancer from benign disease, Rinsho Byori $\mathbf{5 2}$ (2004), 223-230.

[13] M.M. Janković and M.M. Kosanović, Glycosylation of urinary prostate-specific antigen in benign hyperplasia and cancer: assessment by lectin-binding patterns, Clin Biochem 38 (2005), 58-65.
[14] K.M. Slawin, S. Shariat and E. Canto, BPSA: A novel serum marker for benign prostatic hyperplasia, Rev Urol 7 (2005), 52-56.

[15] F.H. Jansen, M. Roobol, G. Jenster, F.H. Schröder and C.H. Bangma, Screening for prostate cancer in 2008 II: the importance of molecular subforms of prostate-specific antigen and tissue kallikreins, Eur Urol 55 (2009), 563-574.

[16] A. Végvári, M. Rezeli, C. Welinder, J. Malm, H. Lilja, G. Marko-Varga and T. Laurell, Identification of prostate-specific antigen (PSA) isoforms in complex biological samples utilizing complementary platforms, J Proteomics 73 (2010), 11371147.

[17] T. Isono, T. Tanaka, S. Kageyama and T. Yoshiki, Structural diversity of cancer-related and non-cancer-related prostatespecific antigen, Clin Chem 48 (2002), 2187-2194.

[18] S.D. Mikolajczyk, L.S. Millar, T.J. Wang, H.G. Rittenhouse, L.S. Marks, W. Song, T.M. Wheeler and K.M. Slawin, A precursor form of prostate-specific antigen is more highly elevated in prostate cancer compared with benign transition zone prostate tissue, Cancer Res 60 (2000), 756-759.

[19] C. Stephan, K. Jung, M. Lein, P. Sinha, D. Schnorr and S.A. Loening, Molecular forms of prostate-specific antigen and human kallikrein 2 as promising tools for early diagnosis of prostate cancer, Cancer Epidemiol Biomarkers Prev 9 (2000), 1133-1147.

[20] S. Prakash and P.W. Robbins, Glycotyping of prostate specific antigen, Glycobiology 10 (2000), 173-176.

[21] T. Okada, Y. Sato, N. Kobayashi, K. Sumida, S. Satomura, S. Matsuura, M. Takasaki and T. Endo, Structural characteristics of the N-glycans of two isoforms of prostate-specific antigens purified from human seminal fluid, Biochim Biophys Acta 1525 (2001), 149-160.

[22] M.M. Kosanović and M.M. Janković, Sialylation and fucosylation of cancer-associated prostate specific antigen, $J$ BUON 10 (2005), 247-250.

[23] G. Tabarés, C.M. Radcliffe, S. Barrabés, M. Ramírez, R.N. Aleixandre, W. Hoesel, R.A. Dwek, P.M. Rudd, R. Peracaula and R. de Llorens, Different glycan structures in prostatespecific antigen from prostate cancer sera in relation to seminal plasma PSA, Glycobiology 16 (2006), 132-145.

[24] G. Tabarés, K. Jung, J. Reiche, C. Stephan, M. Lein, R. Peracaula, R. de Llorens and W. Hösel, Free PSA forms in prostatic tissue and sera of prostate cancer patients: analysis by 2-DE and western blotting of immunopurified samples, Clin Biochem 40 (2007), 343-350.

[25] A. Sarrats, R. Saldova, J. Comet, N. O’Donoghue, R. de Llorens, P.M. Rudd and R. Peracaula, Glycan characterization of PSA 2-DE subforms from serum and seminal plasma, OMICS 14 (2010), 465-474.

[26] M. Tajiri, C. Ohyama and Y. Wada, Oligosaccharide profiles of the prostate specific antigen in free and complexed forms from the prostate cancer patient serum and in seminal plasma: a glycopeptide approach, Glycobiology 18 (2008), 2-8.

[27] A. Sarrats, J. Comet, G. Tabarés, M. Ramírez, R.N. Aleixandre, R. de Llorens and R. Peracaula, Differential percentage of serum prostate-specific antigen subforms suggests a new way to improve prostate cancer diagnosis, Prostate 70 (2010), 1-9.

[28] Z. Chen, H. Chen and T.A. Stamey, Prostate specific antigen in benign prostatic hyperplasia: purification and characterization, J Urol 157 (1997), 2166-2170.

[29] S.D. Mikolajczyk, L.S. Grauer, L.S. Millar, T.M. Hill, A. Kumar, H.G. Rittenhouse, R.L. Wolfert and M.S. Saed, A precursor form of PSA (pPSA) is a component of the free PSA in prostate cancer serum, Urology 50 (1997), 710-714. 
[30] S.D. Mikolajczyk, L.S. Millar, T.J. Wang, H.G. Rittenhouse, R.L. Wolfert, L.S. Marks, W. Song, T.M. Wheeler and K.M. Slawin, BPSA, a specific molecular form of free prostatespecific antigen, is found predominantly in the transition zone of patients with nodular benign prostatic hyperplasia, Urology 55 (2000), 41-45.

[31] T. Tanaka, T. Isono, T. Yoshiki, T. Yuasa and Y. Okada, A novel form of prostate-specific antigen transcript produced by alternative splicing, Cancer Res 60 (2000), 56-59.

[32] V. Kumar, M.I. Hassan, A.K. Singh, S. Dey, T.P. Singh and S. Yadav, Strategy for sensitive and specific detection of molecular forms of PSA based on 2DE and kinetic analysis: a step towards diagnosis of prostate cancer, Clin Chim Acta $\mathbf{4 0 3}$ (2009), 17-22.

[33] World Health Organisation, WHO Laboratory Manual for the Examination of Human Semen and Sperm-Cervical Mucus Interaction, 4th ed., Cambridge, Cambridge University Press, 1999.

[34] M. Merchant and S.R. Weinberger, Recent advancements in surface-enhanced laser desorption/ionization-time of flightmass spectrometry, Electrophoresis 21 (2000), 1164-1177.

[35] T.J. Wang, K.M. Slawin, H.G. Rittenhouse, L.S. Millar and S.D. Mikolajczyk, Benign prostatic hyperplasia-associated prostate-specific antigen (BPSA) shows unique immunoreactivity with anti-PSA monoclonal antibodies, Eur J Biochem 267 (2000), 4040-4045.

[36] J. Peter, C. Unverzagt and W. Hoesel, Analysis of free prostatespecific antigen (PSA) after chemical release from the complex with alpha(1)-antichymotrypsin (PSA-ACT), Clin Chem $\mathbf{4 6}$ (2000), 474-482.

[37] W.D. Bedzy, B. Larsen, S. Gutteridge and R.A. Ballas, Molecular mass determination for prostate-specific antigen and alpha 1-antichymotrypsin complexed in vitro, Biotechnol Appl Biochem 27 (1998), 249-257.

[38] P.S. Basu, M. Ramdhan and S.K. Batabyal, Lectin and serumPSA interaction as a screening test for prostate cancer, Clin Biochem 36 (2003), 373-376. 


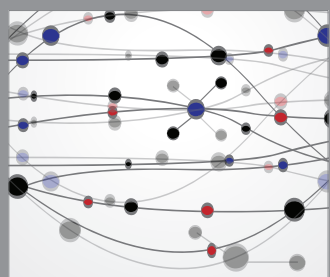

The Scientific World Journal
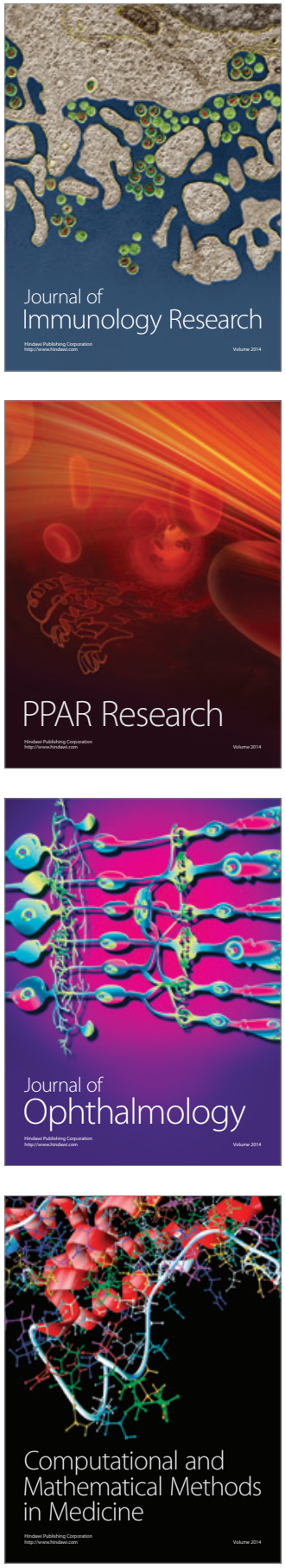

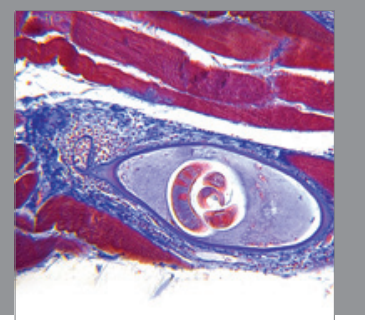

Gastroenterology

Research and Practice
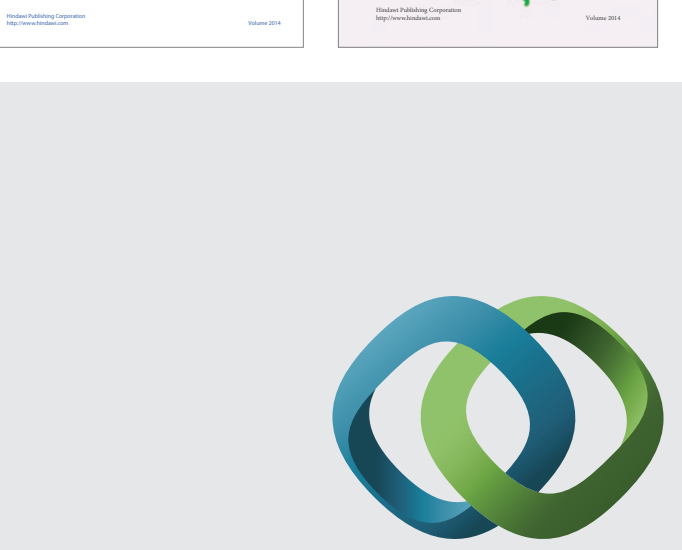

\section{Hindawi}

Submit your manuscripts at

http://www.hindawi.com
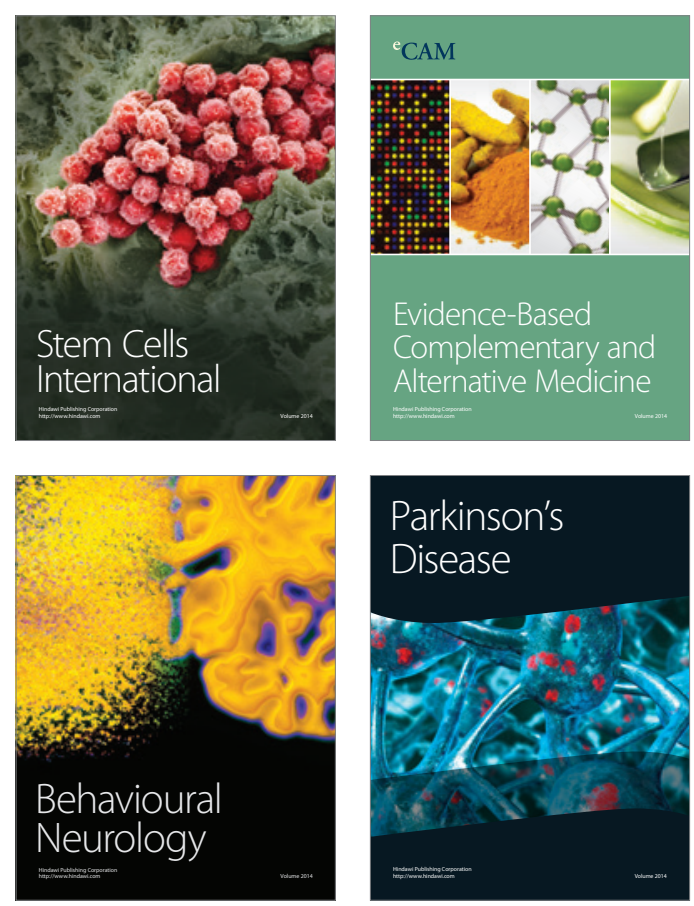

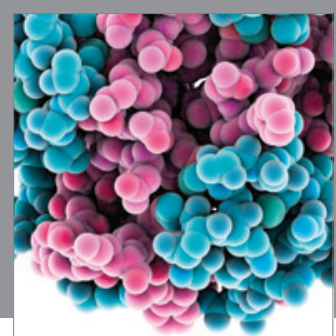

Journal of
Diabetes Research

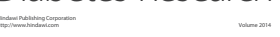

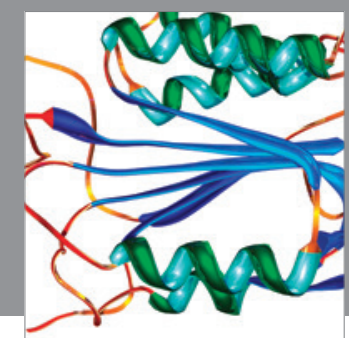

Disease Markers
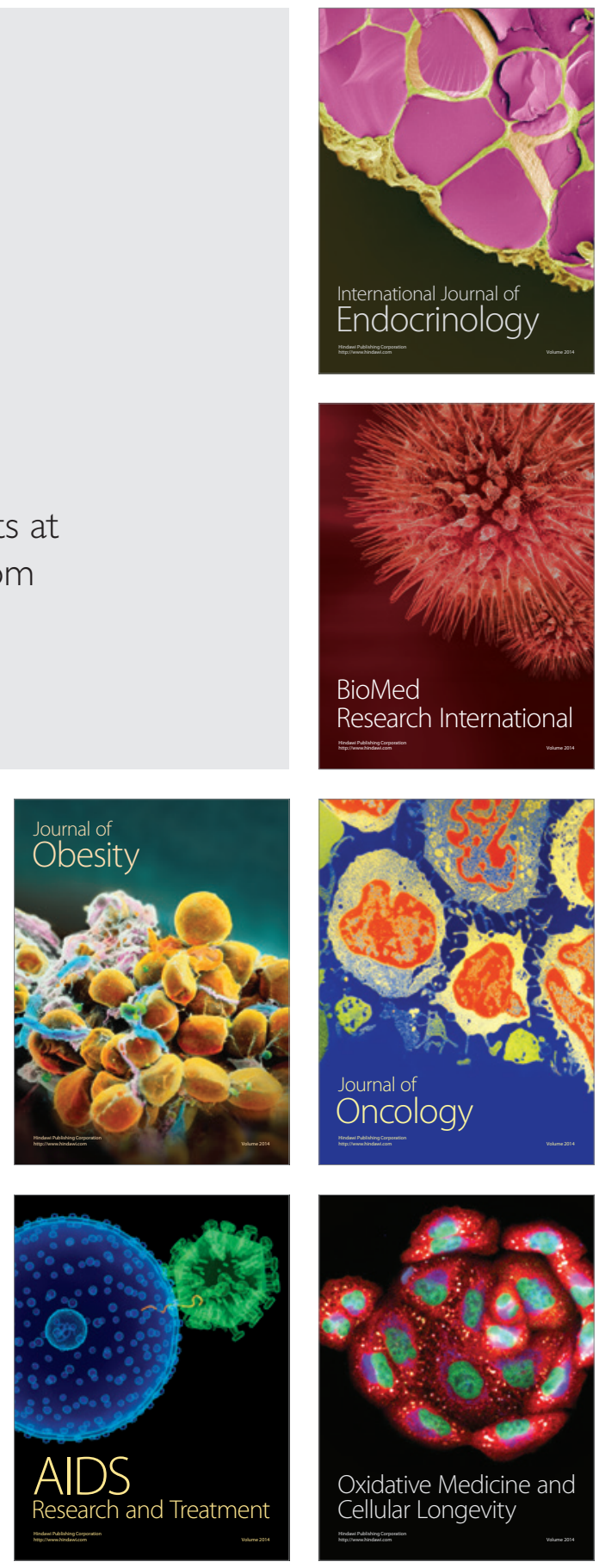